\title{
The Ornstein-Uhlenbeck-Type Model with a Hybrid Dividend Strategy
}

\author{
Dan Zhu and Chuancun Yin \\ School of Mathematical Sciences, Qufu Normal University, Shandong 273165, China \\ Correspondence should be addressed to Chuancun Yin; ccyin@mail.qfnu.edu.cn
}

Received 3 August 2013; Revised 18 December 2013; Accepted 18 December 2013

Academic Editor: Mina Abd-El-Malek

Copyright (C) 2013 D. Zhu and C. Yin. This is an open access article distributed under the Creative Commons Attribution License, which permits unrestricted use, distribution, and reproduction in any medium, provided the original work is properly cited.

\begin{abstract}
We consider the Ornstein-Uhlenbeck-type model. We first introduce the model and then find the ordinary differential equations and boundary conditions satisfied by the dividend functions; closed-form solutions for the dividend value functions are given. We also study the distribution of the time value of ruin. Furthermore, the moments and moment-generating functions of total discounted dividends until ruin are discussed.
\end{abstract}

\section{Introduction}

In recent years, the dividend problem has gained a lot of attention in the actuarial literature. Dividend strategies for insurance risk model were first proposed by de Finetti [1], who considered a discrete time random walk with step size \pm 1 and found that the optimal dividend strategy must be a barrier strategy. From then on, the problem of optimal dividend strategy has been studied in continuous time, for example, Asmussen and Taksar [2], Albrecher et al. [3], Gao and Yin [4], Gerber and Shiu [5, 6], Wan [7] and so on. The optimal dividend problem in a compound Poisson model was studied by Gerber and Shiu [8]. Optimal dividend in an Ornstein-Uhlenbeck-type model with credit and debit interest was considered in Cai et al. [9]. For a class of compound Poisson process perturbed by diffusion with a threshold dividend strategy, the expected discounted penalty function has been studied by Wan [7]. The perturbed Sparre Andersen model with a threshold dividend strategy was settled by Gao and Yin [10].

Recently, the multilayer dividend strategy as an extension of the threshold dividend strategy has drawn many authors attention. For example, the perturbed Sparre Andersen and compound Poisson risk models with multilayer dividend strategy have been studied by Yang and Zhang [11, 12]. The integrodifferential equations for the expected discounted penalty function were derived and solved; when the claims are subexponentially distributed, the asymptotic formula for ruin probability is obtained. The Ornstein-Uhlenbeck-type model is a very important model in applied probability which has recently gained a lot of attention. See, for example, Cai et al. [9] and Fang and Wu [13]. More general, Wong and Zhao [14] consider the optimal dividends and bankruptcy in an Ornstein-Uhlenbeck process with the surplus-dependent credit/debit interest rate. Motivated by the above work, in this paper, we consider a hybrid dividend strategy which combined a barrier strategy with a threshold strategy in an Ornstein-Uhlenbeck-type model. For simplicity, we consider only one threshold and one barrier.

The remainder of the paper is organized as follows. In Section 2, we describe the model and discuss the dividend functions until ruin, in Section 3, we give the limit of dividends level, and in Section 4 we get the expression by Laplace transform of ruin time. The partial differential equation with boundary conditions satisfied by the moments and momentgenerating function is proved in Section 5.

\section{The Model}

Consider the following surplus process:

$$
\mathrm{d} U_{t}=\left(\mu+\rho U_{t}\right) \mathrm{d} t+\sigma \mathrm{d} W_{t}, \quad t \geq 0,
$$

where $\rho>0$ is the force of interest, $\mu>0$ is the drift coefficient, $\sigma>0$ is the diffusion coefficient, and $\left\{W_{t}\right\}_{t \geq 0}$ is the standard Brownian motion. We will assume that 
the company pays dividends according to the following strategy governed by parameters $b_{2}>b_{1}>0$ and $\alpha>$ 0 . Whenever the modified surplus is below the level $b_{1}$, no dividends are paid. However, when the modified surplus is above $b_{1}$ and below the $b_{2}$, dividends are paid continuously at a constant rate $\alpha$. When the modified surplus is above $b_{2}$, dividends are completely paid. For $t \geq 0$, let

$$
D(t)=D_{1}(t)+D_{2}(t)
$$

denote the aggregate dividend paid by time $t$, where $D_{1}(t)$ and $D_{2}(t)$ are caused by the different parts of dividends, respectively. Thus,

$$
\widetilde{U_{t}}=U_{t}-D(t)
$$

is the modified surplus at time $t$. Let $\delta>0$ be the force of interest for valuation; in this paper, we assume that $\rho<\delta$. Let $I(A)$ be the indicator function of event $A$ and let $D$ denote the present value of all dividends until ruin

$$
D=\int_{0}^{T} e^{-\delta t} \mathrm{~d} D_{1}(t)+\int_{0}^{T} e^{-\delta t} \mathrm{~d} D_{2}(t)
$$

where

$$
T=\inf \left\{t \geq 0: \widetilde{U_{t}} \leq 0\right\}
$$

is the time of ruin, and

$$
\begin{gathered}
D_{1}(t)=\alpha \int_{0}^{t} I\left(b_{1} \leq \widetilde{U_{s}} \leq b_{2}\right) \mathrm{d} s, \\
D_{2}(t)=\eta-b_{2}, \\
\eta=\max _{0 \leq s \leq t}\left\{U_{s}-D_{1}(s)\right\} .
\end{gathered}
$$

For $x \geq 0$, we use the symbol $V\left(x ; b_{1}, b_{2}\right)$ to denote the expectation of $D$. That is,

$$
V\left(x ; b_{1}, b_{2}\right)=E\left[D \mid \widetilde{U_{0}}=x\right]=E^{x}[D] .
$$

Define the random times

$$
\begin{gathered}
\tau_{0}^{-}=\inf \left(t \geq 0: U_{t} \leq 0\right), \\
\tau_{b_{1}}^{+}=\inf \left(t \geq 0: U_{t} \geq b_{1}\right), \\
\tau_{b_{1}}^{-}=\inf \left(t \geq 0: \widetilde{U_{t}} \leq b_{1}\right), \\
\tau_{b_{2}}^{+}=\inf \left(t \geq 0: \widetilde{U_{t}} \geq b_{2}\right), \\
\tau=\inf \left(t \geq 0: \widetilde{U_{t}}=b_{1} \text { or } \widetilde{U_{t}}=b_{2}\right),
\end{gathered}
$$

with the convention inf $\emptyset=\infty$.

Lemma 1. Assume that $V(x)$ is twice continuously differentiable on $\left(0, b_{1}\right) \cup\left(b_{1}, b_{2}\right) \cup\left(b_{2}, \infty\right)$. For $0<x \leq b_{1}, V(x)$ satisfies the following ordinary differential equation:

$$
\frac{1}{2} \sigma^{2} V^{\prime \prime}(x)+(\mu+\rho x) V^{\prime}(x)-\delta V(x)=0 ;
$$

for $b_{1}<x \leq b_{2}, V(x)$ satisfies the following ordinary differential equation:

$$
\frac{1}{2} \sigma^{2} V^{\prime \prime}(x)+(\mu+\rho x-\alpha) V^{\prime}(x)-\delta V(x)=0
$$

for $x>b_{2}, V(x)$ satisfies the following equation:

$$
V(x)=x-b_{2}+V\left(b_{2}\right)
$$

with boundary conditions

$$
V\left(0 ; b_{1}, b_{2}\right)=0, \quad V^{\prime}\left(b_{2} ; b_{1}, b_{2}\right)=1 .
$$

Proof. By virtually the same arguments as in Yin and Wen [15], we can prove (9) and (10). The boundary conditions can be derived the same as in Gerber and Shiu [6] or Cai et al. [9].

The ordinary differential equation (9) has two positive independent solutions $f_{1}, f_{2}$ such that $f_{1}$ is strictly decreasing and $f_{2}$ is strictly increasing (see e.g., [16]). Let $f_{3}, f_{4}$ be such solution for the ordinary differential equation (10), where $f_{3}$ is strictly decreasing and $f_{4}$ is strictly increasing. In Cai et al. [9], the authors pointed out that these independent solutions are given by

$$
\begin{aligned}
f_{1}(x)= & \exp \left\{-\frac{1}{\rho \sigma^{2}}(\mu+\rho x)^{2}\right\} \\
& \times U\left(\frac{1}{2}+\frac{\delta}{2 \rho}, \frac{1}{2}, \frac{1}{\rho \sigma^{2}}(\mu+\rho x)^{2}\right), \\
f_{2}(x)= & (\mu+\rho x) \exp \left\{-\frac{1}{\rho \sigma^{2}}(\mu+\rho x)^{2}\right\} \\
& \times M\left(1+\frac{\delta}{2 \rho}, \frac{3}{2}, \frac{1}{\rho \sigma^{2}}(\mu+\rho x)^{2}\right), \\
f_{3}(x)= & \exp \left\{-\frac{1}{\rho \sigma^{2}}(\mu-\alpha+\rho x)^{2}\right\} \\
& \times U\left(\frac{1}{2}+\frac{\delta}{2 \rho}, \frac{1}{2}, \frac{1}{\rho \sigma^{2}}(\mu-\alpha+\rho x)^{2}\right), \\
f_{4}(x)= & (\mu-\alpha+\rho x) \exp \left\{-\frac{1}{\rho \sigma^{2}}(\mu-\alpha+\rho x)^{2}\right\} \\
& \times M\left(1+\frac{\delta}{2 \rho}, \frac{3}{2}, \frac{1}{\rho \sigma^{2}}(\mu-\alpha+\rho x)^{2}\right),
\end{aligned}
$$

where $M$ and $U$ are called the confluent hypergeometric functions of the first and second kind, respectively.

Denote

$$
\begin{gathered}
h(x)=f_{1}(0) f_{2}(x)-f_{2}(0) f_{1}(x), \\
g(x)=f_{3}\left(b_{2}\right) f_{4}(x)-f_{4}\left(b_{2}\right) f_{3}(x), \\
k(x)=f_{3}(x) f_{4}\left(b_{1}\right)-f_{3}\left(b_{1}\right) f_{4}(x) .
\end{gathered}
$$

The expressions of the expected discounted dividend payments are given by Theorem 2 . 
Theorem 2. (i) For $0 \leq x \leq b_{1}$,

$$
\begin{gathered}
V\left(x ; b_{1}, b_{2}\right) \\
=\left(\left(-\alpha g^{\prime}\left(b_{1}\right) k^{\prime}\left(b_{2}\right)+k^{\prime}\left(b_{1}\right) g\left(b_{1}\right) \delta+\alpha k^{\prime}\left(b_{1}\right) g^{\prime}\left(b_{1}\right)\right)\right. \\
\times\left(\delta \left[h^{\prime}\left(b_{1}\right) g\left(b_{1}\right) k^{\prime}\left(b_{2}\right)-g^{\prime}\left(b_{1}\right) h\left(b_{1}\right) k^{\prime}\left(b_{2}\right)\right.\right. \\
\left.\left.\left.+k^{\prime}\left(b_{1}\right) g^{\prime}\left(b_{1}\right) h\left(b_{1}\right)\right]\right)^{-1}\right) h(x) .
\end{gathered}
$$

(ii) For $b_{1}<x \leq b_{2}$,

$$
\begin{aligned}
& V\left(x ; b_{1}, b_{2}\right) \\
& =\frac{\alpha}{\delta}+\frac{k(x)}{k^{\prime}\left(b_{2}\right)} \\
& +\left(\left[\delta h\left(b_{1}\right) k^{\prime}\left(b_{1}\right)-\alpha h^{\prime}\left(b_{1}\right) k^{\prime}\left(b_{2}\right)\right]\right. \\
& \left.\quad \times\left[\left(g(x) k^{\prime}\left(b_{2}\right)-g^{\prime}\left(b_{2}\right) k(x)\right)\right]\right) \\
& \times\left(k ^ { \prime } ( b _ { 2 } ) \delta \left[h^{\prime}\left(b_{1}\right) g\left(b_{1}\right) k^{\prime}\left(b_{2}\right)-g^{\prime}\left(b_{1}\right) h\left(b_{1}\right) k^{\prime}\left(b_{2}\right)\right.\right. \\
& \left.\left.+k^{\prime}\left(b_{1}\right) g^{\prime}\left(b_{1}\right) h\left(b_{1}\right)\right]\right)^{-1} .
\end{aligned}
$$

Proof. When $0<x \leq b_{1}$, by the strong Markov property of the process $U_{t}$, we have

$$
\begin{aligned}
E^{x}[D] & =E^{x}\left[D I\left(\tau_{b_{1}}^{+}<\tau_{0}^{-}\right)\right]+E^{x}\left[D I\left(\tau_{b_{1}}^{+}>\tau_{0}^{-}\right)\right] \\
& =E^{x}\left[D I\left(\tau_{b_{1}}^{+}<\tau_{0}^{-}\right)\right] \\
& =E^{x}\left[e^{-\delta \tau_{b_{1}^{+}} I}\left(\tau_{b_{1}}^{+}<\tau_{0}^{-}\right)\right] V\left(b_{1} ; b_{1}, b_{2}\right) .
\end{aligned}
$$

For $0<x \leq b_{1}$, using Itô's formula or Dynkin's formula as in Li et al. [17], we find that $f(x):=E^{x}\left[e^{-\delta \tau_{b_{1}^{+}}} I\left(\tau_{b_{1}}^{+}<\tau_{0}^{-}\right)\right]$ satisfies the ordinary differential equation (9), with boundary conditions $f\left(b_{1}\right)=1, f(0)=0$. Assume that the solution of the equation is $f(x)=c_{1} f_{1}(x)+c_{2} f_{2}(x)$; from the boundary conditions, we obtain

$$
\begin{aligned}
& c_{1}=\frac{-f_{2}(0)}{f_{1}(0) f_{2}\left(b_{1}\right)-f_{2}(0) f_{1}\left(b_{1}\right)}, \\
& c_{2}=\frac{f_{1}(0)}{f_{1}(0) f_{2}\left(b_{1}\right)-f_{2}(0) f_{1}\left(b_{1}\right)} .
\end{aligned}
$$

So we have

$$
E^{x}\left[e^{-\delta \tau_{b_{1}^{+}}} I\left(\tau_{b_{1}}^{+}<\tau_{0}^{-}\right)\right]=\frac{h(x)}{h\left(b_{1}\right)} .
$$

Similarly, when $b_{1}<x \leq b_{2}$,

$$
\begin{aligned}
E^{x}[D]= & E^{x}\left[\int_{0}^{\tau} \alpha e^{-\delta t} d t\right]+E^{x}\left[e^{-\delta t}\right] V\left(\widetilde{U}_{\tau} ; b_{1}, b_{2}\right) \\
= & E^{x}\left[\left(-\frac{\alpha}{\delta}\right)\left(e^{-\delta \tau}-1\right)\right] \\
& +E^{x}\left[e^{-\delta \tau} I\left(\tau_{b_{1}}^{-}<\tau_{b_{2}}^{+}\right)\right] V\left(b_{1} ; b_{1}, b_{2}\right) \\
& +E^{x}\left[e^{-\delta \tau} I\left(\tau_{b_{2}}^{+}<\tau_{b_{1}}^{-}\right)\right] V\left(b_{2} ; b_{1}, b_{2}\right) .
\end{aligned}
$$

For $b_{1}<x \leq b_{2}$, using Itô’s formula or Dynkin's formula, we have that $f(x):=E^{x}\left[e^{-\delta \tau_{b_{1}}^{-}} I\left(\tau_{b_{1}}^{-}<\tau_{b_{2}}^{+}\right)\right]$satisfies the ordinary differential equation (10) with boundary conditions $f\left(b_{1}\right)=1, f\left(b_{2}\right)=0$. Assume that the solution of the equation is $f(x)=c_{3} f_{3}(x)+c_{4} f_{4}(x)$; from the boundary conditions, we obtain

$$
\begin{aligned}
& c_{3}=\frac{-f_{4}\left(b_{2}\right)}{f_{3}\left(b_{2}\right) f_{4}\left(b_{1}\right)-f_{4}\left(b_{2}\right) f_{3}\left(b_{1}\right)}, \\
& c_{4}=\frac{f_{3}\left(b_{2}\right)}{f_{3}\left(b_{2}\right) f_{4}\left(b_{1}\right)-f_{4}\left(b_{2}\right) f_{3}\left(b_{1}\right)},
\end{aligned}
$$

so we have

$$
E^{x}\left[e^{-\delta \tau_{b_{1}}^{-}} I\left(\tau_{b_{1}}^{-}<\tau_{b_{2}}^{+}\right)\right]=\frac{g(x)}{g\left(b_{1}\right)} .
$$

Similarly, $f(x):=E^{x}\left[e^{-\delta \tau_{b_{2}}^{+}} I\left(\tau_{b_{2}}^{+}<\tau_{b_{1}}^{-}\right)\right]$satisfies (10) with boundary conditions $f\left(b_{1}\right)=0, f\left(b_{2}\right)=1$, so we get

$$
\begin{aligned}
E^{x}\left[e^{-\delta \tau_{b_{2}}^{+}} I\left(\tau_{b_{2}}^{+}<\tau_{b_{1}}^{-}\right)\right] & =\frac{f_{3}(x) f_{4}\left(b_{1}\right)-f_{3}\left(b_{1}\right) f_{4}(x)}{g\left(b_{1}\right)} \\
& =\frac{k(x)}{g\left(b_{1}\right)},
\end{aligned}
$$

where

$$
g\left(b_{1}\right)=k\left(b_{2}\right) .
$$

For $0<x \leq b_{1}$, dividends will be payable if the surplus process without ruin and reaches $b_{1}$, so that

$$
\begin{aligned}
V\left(x ; b_{1}, b_{2}\right) & =E^{x}\left[e^{-\delta \tau_{b_{1}^{+}}} I\left(\tau_{b_{1}}^{+}<\tau_{0}^{-}\right)\right] V\left(b_{1} ; b_{1}, b_{2}\right) \\
& =\frac{h(x)}{h\left(b_{1}\right)} V\left(b_{1} ; b_{1}, b_{2}\right) .
\end{aligned}
$$


For $b_{1}<x \leq b_{2}$, dividends are paid continuously at a constant rate $\alpha$, so we obtain

$$
\begin{aligned}
V\left(x ; b_{1}, b_{2}\right)= & \frac{\alpha}{\delta}+E^{x}\left[e^{-\delta \tau} I\left(\tau_{b_{1}}^{-}<\tau_{b_{2}}^{+}\right)\right]\left(V\left(b_{1} ; b_{1}, b_{2}\right)-\frac{\alpha}{\delta}\right) \\
& +E^{x}\left[e^{-\delta \tau} I\left(\tau_{b_{2}}^{+}<\tau_{b_{1}}^{-}\right)\right]\left(V\left(b_{2} ; b_{1}, b_{2}\right)-\frac{\alpha}{\delta}\right) \\
= & \frac{\alpha}{\delta}+\frac{g(x)}{g\left(b_{1}\right)}\left(V\left(b_{1} ; b_{1}, b_{2}\right)-\frac{\alpha}{\delta}\right) \\
& +\frac{k(x)}{g\left(b_{1}\right)}\left(V\left(b_{2} ; b_{1}, b_{2}\right)-\frac{\alpha}{\delta}\right) .
\end{aligned}
$$

Using $V^{\prime}\left(b_{2} ; b_{1}, b_{2}\right)=1$ and $V^{\prime}\left(b_{1}+; b_{1}, b_{2}\right)=V^{\prime}\left(b_{1}-; b_{1}\right.$, $b_{2}$ ), we get

$$
\begin{aligned}
\frac{g^{\prime}\left(b_{2}\right)}{g\left(b_{1}\right)}\left(V\left(b_{1} ; b_{1}, b_{2}\right)-\frac{\alpha}{\delta}\right) & +\frac{k^{\prime}\left(b_{2}\right)}{g\left(b_{1}\right)}\left(V\left(b_{2} ; b_{1}, b_{2}\right)-\frac{\alpha}{\delta}\right)=1, \\
\frac{h^{\prime}\left(b_{1}\right)}{h\left(b_{1}\right)} V\left(b_{1} ; b_{1}, b_{2}\right)= & \frac{g^{\prime}\left(b_{1}\right)}{g\left(b_{1}\right)}\left(V\left(b_{1} ; b_{1}, b_{2}\right)-\frac{\alpha}{\delta}\right) \\
& +\frac{k^{\prime}\left(b_{1}\right)}{g\left(b_{1}\right)}\left(V\left(b_{2} ; b_{1}, b_{2}\right)-\frac{\alpha}{\delta}\right) .
\end{aligned}
$$

With some careful calculations, we obtain $V\left(b_{1} ; b_{1}, b_{2}\right)$ and $V\left(b_{2} ; b_{1}, b_{2}\right)$, so we get the results (18) and (19).

\section{The Special Dividends Strategy}

In this section, we consider the limit of dividends level. Let $b_{2} \rightarrow \infty$; by the expressions of $k$ and $g$, we have

$$
\begin{aligned}
& g\left(b_{1}\right) \sim-f_{3}\left(b_{1}\right) f_{4}\left(b_{2}\right), \\
& g^{\prime}\left(b_{1}\right) \sim-f^{\prime}\left(b_{1}\right) f_{4}\left(b_{2}\right), \\
& k^{\prime}\left(b_{2}\right) \sim-f_{3}\left(b_{1}\right) f_{4}^{\prime}\left(b_{2}\right),
\end{aligned}
$$

where

$$
f_{4}\left(b_{2}\right) \longrightarrow \infty, \quad f_{4}^{\prime}\left(b_{2}\right) \longrightarrow \infty .
$$

Substituting the above expressions into (18) and (19), and setting

$$
\begin{gathered}
A=-\alpha f_{3}^{\prime}\left(b_{1}\right) f_{4}\left(b_{2}\right) f_{3}\left(b_{1}\right) f_{4}^{\prime}\left(b_{2}\right), \\
B=\delta k^{\prime}\left(b_{1}\right) f_{3}\left(b_{1}\right) f_{4}\left(b_{2}\right), \\
C=\alpha k^{\prime}\left(b_{1}\right) f_{3}^{\prime}\left(b_{1}\right) f_{4}\left(b_{2}\right), \\
D=h^{\prime}\left(b_{1}\right) f_{3}\left(b_{1}\right) f_{4}^{\prime}\left(b_{2}\right) f_{3}\left(b_{1}\right) f_{4}\left(b_{2}\right), \\
E=f_{3}^{\prime}\left(b_{1}\right) f_{4}\left(b_{2}\right) f_{3}\left(b_{1}\right) f_{4}^{\prime}\left(b_{2}\right), \\
F=f_{3}^{\prime}\left(b_{1}\right) f_{4}\left(b_{2}\right) k^{\prime}\left(b_{1}\right) h\left(b_{1}\right),
\end{gathered}
$$

we obtain, for $0<x \leq b_{1}$,

$$
V\left(x ; b_{1}, b_{2}\right)=\frac{A-B-C}{\delta[D-E-F]} h(x) .
$$

Let

$$
\begin{gathered}
A^{\prime}=\delta h\left(b_{1}\right) k^{\prime}\left(b_{1}\right)+\alpha h^{\prime}\left(b_{1}\right) f_{3}\left(b_{1}\right) f_{4}^{\prime}\left(b_{2}\right), \\
B^{\prime}=-\left(g(x) f_{3}\left(b_{1}\right) f_{4}^{\prime}\left(b_{2}\right)-g^{\prime}\left(b_{2}\right) k(x)\right), \\
C^{\prime}=h^{\prime}\left(b_{1}\right) f_{3}^{2}\left(b_{1}\right) f_{4}\left(b_{2}\right) f_{4}^{\prime}\left(b_{2}\right), \\
D^{\prime}=k^{\prime}\left(b_{1}\right) f_{4}\left(b_{2}\right) h\left(b_{1}\right) f_{3}\left(b_{1}\right) f_{4}^{\prime}\left(b_{2}\right), \\
E^{\prime}=k^{\prime 2}\left(b_{1}\right) f_{4}\left(b_{2}\right) h\left(b_{1}\right) ;
\end{gathered}
$$

we obtain, for $b_{1}<x \leq \infty$,

$$
\begin{aligned}
V\left(x ; b_{1}, b_{2}\right)= & \frac{\alpha}{\delta}+\frac{k(x)}{-f_{3}\left(b_{1}\right) f_{4}^{\prime}\left(b_{2}\right)} \\
& -\frac{A^{\prime} B^{\prime}}{f_{3}\left(b_{1}\right) f_{4}^{\prime}\left(b_{2}\right) \delta\left[C^{\prime}-D^{\prime}-E^{\prime}\right]} .
\end{aligned}
$$

Then dividing numerator and denominator of (34) and (36) by $f_{4}\left(b_{2}\right) f_{4}^{\prime}\left(b_{2}\right)$, we get the expected discounted dividend payments for the threshold strategy

$V(x ; b)$

$$
= \begin{cases}\frac{\alpha}{\delta} \frac{f_{3}^{\prime}(b) h(x)}{f_{3}^{\prime}(b) h(b)-f_{3}(b) h^{\prime}(b)}, & \text { if } 0<x \leq b_{1}, \\ \frac{\alpha}{\delta}+\frac{\alpha}{\delta} \frac{f_{3}(x) h^{\prime}(b)}{f_{3}^{\prime}(b) h(b)-f_{3}(b) h^{\prime}(b)}, & \text { if } x>b_{2},\end{cases}
$$

which is (15) in Fang and Wu [13].

Remark 3. Similarly, when $b_{1}=b_{2}=b$, we get the expected discounted dividend payments for the barrier strategy

$$
V(x ; b)= \begin{cases}\frac{h(x)}{h^{\prime}(b)}, & \text { if } 0<x \leq b, \\ x-b+\frac{h(b)}{h^{\prime}(b)}, & \text { if } x>b,\end{cases}
$$

which is (9) and (10) in Cai et al. [9].

\section{The Time Value of Ruin under a Hybrid Dividend Strategy}

In this section, we focus on the Laplace transform of the time value of ruin. We assume that dividends are paid according to threshold strategy with parameters $b_{1}, \alpha$ and barrier strategy with parameter $b_{2}$. Let $L\left(x ; b_{1}, b_{2}\right)$ denote the Laplace transform of the time value of ruin; for $x \geq 0$,

$$
L\left(x ; b_{1}, b_{2}\right)=E\left[e^{-\delta T} \mid U_{0}=x\right] .
$$

Let

$$
\rho(x)=f_{1}(x) f_{2}\left(b_{1}\right)-f_{2}(x) f_{1}\left(b_{1}\right) .
$$


Theorem 4. For $0<x \leq b_{1}$, one has

$$
L\left(x ; b_{1}, b_{2}\right)=\frac{h(x)}{h\left(b_{1}\right)} A+\frac{\rho(x)}{h\left(b_{1}\right)},
$$

and, for $b_{1}<x \leq b_{2}$, one has

$$
L\left(x ; b_{1}, b_{2}\right)=\frac{g(x)}{g\left(b_{1}\right)} A+\frac{k(x)}{g\left(b_{1}\right)} B,
$$

where

$$
\begin{aligned}
A= & \left(k^{\prime}\left(b_{1}\right) h\left(b_{1}\right) g\left(b_{1}\right)-\rho^{\prime}\left(b_{1}\right) k^{\prime}\left(b_{2}\right) g\left(b_{1}\right)\right) \\
& \times\left(g^{\prime}\left(b_{2}\right) k^{\prime}\left(b_{1}\right) h\left(b_{1}\right)+g\left(b_{1}\right) h^{\prime}\left(b_{1}\right) k^{\prime}\left(b_{2}\right)\right. \\
& \left.\quad-g^{\prime}\left(b_{1}\right) h\left(b_{1}\right) k^{\prime}\left(b_{2}\right)\right)^{-1}, \\
B= & \left(g^{2}\left(b_{1}\right) h^{\prime}\left(b_{1}\right)-g\left(b_{1}\right) g^{\prime}\left(b_{1}\right) h\left(b_{1}\right)\right. \\
& \left.+g\left(b_{1}\right) g^{\prime}\left(b_{2}\right) \rho^{\prime}\left(b_{1}\right)\right) \\
& \times\left(g^{\prime}\left(b_{2}\right) k^{\prime}\left(b_{1}\right) h\left(b_{1}\right)+g\left(b_{1}\right) h^{\prime}\left(b_{1}\right) k^{\prime}\left(b_{2}\right)\right. \\
& \left.\quad-g^{\prime}\left(b_{1}\right) h\left(b_{1}\right) k^{\prime}\left(b_{2}\right)\right)^{-1} .
\end{aligned}
$$

Proof. For $0<x \leq b_{1}$, applying the strong Markov property, we obtain

$$
\begin{aligned}
L\left(x ; b_{1}, b_{2}\right)= & E^{x}\left[e^{-\delta T}\right] \\
= & E^{x}\left[e^{-\delta T} I\left(\tau_{b_{1}}^{+}<\tau_{0}^{-}\right)\right]+E^{x}\left[e^{-\delta T} I\left(\tau_{b_{1}}^{+}>\tau_{0}^{-}\right)\right] \\
= & E^{x}\left[e^{-\delta \tau_{b_{1}}^{+}} I\left(\tau_{b_{1}}^{+}<\tau_{0}^{-}\right)\right] L\left(b_{1} ; b_{1}, b_{2}\right) \\
& +E^{x}\left[e^{-\delta \tau_{0}^{-}} I\left(\tau_{b_{1}}^{+}>\tau_{0}^{-}\right)\right] \\
= & \frac{h(x)}{h\left(b_{1}\right)} L\left(b_{1} ; b_{1}, b_{2}\right)+\frac{\rho(x)}{h\left(b_{1}\right)} .
\end{aligned}
$$

Similarly, for $b_{1}<x \leq b_{2}$,

$$
\begin{aligned}
L\left(x ; b_{1}, b_{2}\right)= & E^{x}\left[e^{-\delta T}\right] \\
= & E^{x}\left[e^{-\delta \tau}\right] L\left(x(\tau) ; b_{1}, b_{2}\right) \\
= & E^{x}\left[e^{-\delta \tau} I\left(\tau_{b_{1}}^{-}<\tau_{b_{2}}^{+}\right)\right] L\left(b_{1} ; b_{1}, b_{2}\right) \\
& +E^{x}\left[e^{-\delta \tau} I\left(\tau_{b_{1}}^{-}>\tau_{b_{2}}^{+}\right)\right] L\left(b_{2} ; b_{1}, b_{2}\right) \\
= & \frac{g(x)}{g\left(b_{1}\right)} L\left(b_{1} ; b_{1}, b_{2}\right)+\frac{k(x)}{g\left(b_{1}\right)} L\left(b_{2} ; b_{1}, b_{2}\right) .
\end{aligned}
$$

It can be verified that $L^{\prime}\left(b_{2} ; b_{1}, b_{2}\right)=1$ and $L^{\prime}\left(b_{1}+; b_{1}, b_{2}\right)=$ $L^{\prime}\left(b_{1}-; b_{1}, b_{2}\right)$, so we get

$$
\begin{aligned}
\frac{g^{\prime}\left(b_{2}\right)}{g\left(b_{1}\right)} L\left(b_{1} ; b_{1}, b_{2}\right)+\frac{k^{\prime}\left(b_{2}\right)}{g\left(b_{1}\right)} L\left(b_{2} ; b_{1}, b_{2}\right)=1, & \\
\frac{h^{\prime}\left(b_{1}\right)}{h\left(b_{1}\right)} L\left(b_{1} ; b_{1}, b_{2}\right)+\frac{\rho^{\prime}(x)}{h\left(b_{1}\right)}= & \frac{g^{\prime}\left(b_{1}\right)}{g\left(b_{1}\right)} L\left(b_{1} ; b_{1}, b_{2}\right) \\
& +\frac{k^{\prime}\left(b_{1}\right)}{g\left(b_{1}\right)} L\left(b_{2} ; b_{1}, b_{2}\right) .
\end{aligned}
$$

From $(46)$ we obtain $L\left(b_{1} ; b_{1}, b_{2}\right)$ and $L\left(b_{2} ; b_{1}, b_{2}\right)$, so we get the results (41) and (42).

Remark 5. (1) Let $\phi\left(x ; b_{1}, b_{2}\right)$ denote the expected discounted penalty at ruin; in this model, the penalty at ruin is a constant $M>0$, so we get

$$
\phi\left(x ; b_{1}, b_{2}\right)=M L\left(x ; b_{1}, b_{2}\right) .
$$

Substitution of (41) and (42) into (47) yields an expression for $\phi\left(x ; b_{1}, b_{2}\right)$.

(2) Let $\psi\left(x ; b_{1}, b_{2}\right)=P\left(T<\infty \mid U_{0}=x\right)$ be the probability of ruin. Note that

$$
\begin{aligned}
E\left[e^{-\delta T} \mid U_{0}=x\right]= & E^{x}\left[e^{-\delta T} I(T<\infty)\right] \\
& +E^{x}\left[e^{-\delta T} I(T=\infty)\right] \\
= & E^{x}\left[e^{-\delta T} I(T<\infty)\right] ;
\end{aligned}
$$

then

$$
\psi\left(x ; b_{1}, b_{2}\right)=\lim _{\delta \rightarrow 0} L\left(x ; b_{1}, b_{2}\right)=P(T<\infty) .
$$

From (14)-(18), we have

$$
\begin{aligned}
\lim _{\delta \rightarrow 0} f_{1}(x)= & \exp \left\{-\frac{1}{\rho \sigma^{2}}(\mu+\rho x)^{2}\right\} \\
& \times U\left(\frac{1}{2}, \frac{1}{2}, \frac{1}{\rho \sigma^{2}}(\mu+\rho x)^{2}\right), \\
\lim _{\delta \rightarrow 0} f_{2}(x)= & (\mu+\rho x) \exp \left\{-\frac{1}{\rho \sigma^{2}}(\mu+\rho x)^{2}\right\} \\
& \times M\left(1, \frac{3}{2}, \frac{1}{\rho \sigma^{2}}(\mu+\rho x)^{2}\right), \\
\lim _{\delta \rightarrow 0} f_{3}(x)= & \exp \left\{-\frac{1}{\rho \sigma^{2}}(\mu-\alpha+\rho x)^{2}\right\} \\
& \times U\left(\frac{1}{2}, \frac{1}{2}, \frac{1}{\rho \sigma^{2}}(\mu-\alpha+\rho x)^{2}\right), \\
\lim _{\delta \rightarrow 0} f_{4}(x)= & (\mu-\alpha+\rho x) \exp \left\{-\frac{1}{\rho \sigma^{2}}(\mu-\alpha+\rho x)^{2}\right\} \\
& \times M\left(1, \frac{3}{2}, \frac{1}{\rho \sigma^{2}}(\mu-\alpha+\rho x)^{2}\right) .
\end{aligned}
$$

Taking limit in (41) and (42) yields $\psi\left(x ; b_{1}, b_{2}\right)$. 


\section{The Moment-Generating Function of $D$}

In this section, the moment-generating function of the hybrid dividend payments is discussed. We adopt a similar approach to that of Gao and Yin in Section 3 [10].

Let

$$
M\left(x, y ; b_{1}, b_{2}\right)=E\left[e^{y D} \mid U_{0}=x\right]
$$

denote the moment-generating function of $D$, and let

$$
V_{m}\left(x ; b_{1}, b_{2}\right)=E\left[D^{m} U_{0}=x\right], \quad m=1,2,3, \ldots,
$$

denote the $m$ th moment function. The following theorem provides differential equations for the function $M\left(x, y ; b_{1}\right.$, $\left.b_{2}\right)$.

Theorem 6. The moment-generating function $M\left(x, y ; b_{1}, b_{2}\right)$ satisfies the partial differential equations

$$
\begin{gathered}
\frac{\sigma^{2}}{2} \frac{\partial^{2} M}{\partial x^{2}}+\frac{\partial M}{\partial x}(\rho x+\mu)-\frac{\partial M}{\partial y} y \delta=0, \quad 0<x \leq b_{1}, \\
\frac{\sigma^{2}}{2} \frac{\partial^{2} M}{\partial x^{2}}+\frac{\partial M}{\partial x}(\rho x+\mu-\alpha)-\frac{\partial M}{\partial y} y \delta=0, \quad b_{1}<x \leq b_{2},
\end{gathered}
$$

with boundary conditions

$$
M\left(0, y ; b_{1}, b_{2}\right)=1, \quad \lim _{x \rightarrow \infty} M\left(x, y ; b_{1}, b_{2}\right)=e^{y \alpha / \delta} .
$$

Proof. (i) We first provide the solution of $U_{t}$. Consider the SDE

$$
\mathrm{d} U_{t}=\left(\mu+\rho U_{t}\right) \mathrm{d} t+\sigma \mathrm{d} W_{t} ;
$$

note that, in the case $\sigma=0$, the solution to the ODE is $(1 / \rho)\left(\rho U_{0}+\mu\right) e^{\rho t}-\mu / \rho$. To solve the SDE, consider the process

$$
Y(t)=\frac{1}{\rho}\left(\rho U_{0}+\mu\right) e^{-\rho t}-\frac{\mu}{\rho} .
$$

Let $P(t)=U_{t} e^{-\rho t}$ and $Q(t)=(\mu / \rho)\left(e^{-\rho t}-1\right)$, so we have $d Y(t)=d P(t)+d Q(t)$,

$$
\begin{aligned}
d P(t) & =d U_{t} e^{-\rho t} \\
& =U_{t}(-\rho) e^{-\rho t} d t+e^{-\rho t} d U_{t}+d\left[U_{t}, e^{-\rho t}\right] \\
& =U_{t}(-\rho) e^{-\rho t} d t+e^{-\rho t} d U_{t} \\
& =U_{t}(-\rho) e^{-\rho t} d t+e^{-\rho t}\left(\mu+\rho U_{t}\right) d t+\sigma \mathrm{d} W_{t} \\
& =e^{-\rho t} \mu d t+e^{-\rho t} \sigma \mathrm{d} W_{t}, \\
& d Q(t)=-e^{-\rho t} \mu d t ;
\end{aligned}
$$

we get $d Y(t)=e^{-\rho t} \sigma \mathrm{d} W_{t}$, so we obtain

$$
Y(t)=Y(0)+\int_{0}^{t} e^{-\rho s} \sigma \mathrm{d} W_{s} .
$$

Now the solution for $U_{t}$ is

$$
U_{t}=e^{\rho t}\left\{U_{0}-\frac{\mu}{\rho}\left(e^{-\rho t}-1\right)+\int_{0}^{t} e^{-\rho s} \sigma \mathrm{d} W_{s}\right\} .
$$

(ii) Now we derive the integrodifferential equations for $M\left(x, y ; b_{1}, b_{2}\right)$.

For $0<x \leq b_{1}$, we consider a small time interval $[0, t]$, the time $t>0$ such that the surplus will not reach $b_{1}$ before $t$; in view of the strong Markov property of the surplus process $\left\{U_{t}, t \geq 0\right\}$, we have

$$
M\left(x, y ; b_{1}, b_{2}\right)=E\left[M\left(U_{t}, y e^{-\delta t} ; b_{1}, b_{2}\right)\right]+o(t) .
$$

By Taylor expansion, we have

$$
\begin{aligned}
E & {\left[M\left(U_{t}, y e^{-\delta t} ; b_{1}, b_{2}\right)\right] } \\
= & E\left[M\left(x, y ; b_{1}, b_{2}\right)\right]+\frac{\partial M}{\partial x}\left(U_{t}-x\right)+\frac{\partial M}{\partial y}\left(y e^{-\delta t}-y\right) \\
& +\frac{1}{2}\left(\frac{\partial^{2} M}{\partial x^{2}}\left(U_{t}-x\right)^{2} \frac{\partial^{2} M}{\partial y^{2}}\left(y e^{-\delta t}-y\right)^{2}\right) \\
& +\frac{\partial M}{\partial x \partial y}\left(U_{t}-x\right)\left(y e^{-\delta t}-y\right)+o(t) .
\end{aligned}
$$

Subtracting $M\left(x, y ; b_{1}, b_{2}\right)$ from each side of the above equation, dividing by $t$, and then letting $t \rightarrow 0$, we achieve

$$
\frac{\sigma^{2}}{2} \frac{\partial^{2} M}{\partial x^{2}}+\frac{\partial M}{\partial x} \rho\left(x+\frac{\mu}{\rho}\right)-\frac{\partial M}{\partial y} y \delta=0
$$

Similarly, for $b_{1}<x \leq b_{2}$, we consider a small time interval $[0, t]$, the time $t>0$ such that the surplus will not reach $b_{2}$; we have

$$
M\left(x, y ; b_{1}, b_{2}\right)=E\left[M\left(U_{t}-\alpha t, y e^{-\delta t} ; b_{1}, b_{2}\right)\right]+o(t) .
$$

By Taylor expansion, we get

$$
\begin{aligned}
E[ & \left.M\left(U_{t}-\alpha t, y e^{-\delta t} ; b_{1}, b_{2}\right)\right] \\
= & E\left[M\left(x, y ; b_{1}, b_{2}\right)\right]+\frac{\partial M}{\partial x}\left(U_{t}-x-\alpha t\right) \\
& +\frac{\partial M}{\partial y}\left(y e^{-\delta t}-y\right) \\
& +\frac{1}{2}\left(\frac{\partial^{2} M}{\partial x^{2}}\left(U_{t}-x-\alpha t\right)^{2} \frac{\partial^{2} M}{\partial y^{2}}\left(y e^{-\delta t}-y\right)^{2}\right) \\
& +\frac{\partial M}{\partial x \partial y}\left(U_{t}-x-\alpha t\right)\left(y e^{-\delta t}-y\right)+o(t) .
\end{aligned}
$$

Subtracting $M\left(x, y ; b_{1}, b_{2}\right)$ from each side of (63), dividing by $t$, and then letting $t \rightarrow 0$, we achieve

$$
\frac{\sigma^{2}}{2} \frac{\partial^{2} M}{\partial x^{2}}+\frac{\partial M}{\partial x}(\rho x+\mu-\alpha)-\frac{\partial M}{\partial y} y \delta=0 .
$$

The proof of boundary conditions is routine. This ends the proof of Theorem 6 . 
Theorem 7. For $0<x \leq b_{1}, V_{m}\left(x ; b_{1}, b_{2}\right)$ satisfies

$$
\begin{aligned}
& \frac{\sigma^{2}}{2} V_{m}^{\prime \prime}\left(x ; b_{1}, b_{2}\right)+(\rho x+\rho) V_{m}^{\prime}\left(x ; b_{1}, b_{2}\right) \\
& \quad-\delta m V_{m}\left(x ; b_{1}, b_{2}\right)=0,
\end{aligned}
$$

and, for $b_{1}<x \leq b_{2}, V_{m}\left(x ; b_{1}, b_{2}\right)$ satisfies

$$
\begin{aligned}
& \frac{\sigma^{2}}{2} V_{m}^{\prime \prime}\left(x ; b_{1}, b_{2}\right)+(\rho x+\rho-\alpha) V_{m}^{\prime}\left(x ; b_{1}, b_{2}\right) \\
& \quad-\delta m V_{m}\left(x ; b_{1}, b_{2}\right)=0,
\end{aligned}
$$

with the boundary conditions

$$
V_{m}\left(0 ; b_{1}, b_{2}\right)=0,\left.V_{m}^{\prime}\left(x ; b_{1}, b_{2}\right)\right|_{x=b_{2}}=m V_{m-1}\left(b_{2} ; b_{1}, b_{2}\right) \text {. }
$$

Proof. Recall that $M\left(x, y ; b_{1}, b_{2}\right)=E\left[e^{y D}\right]$, and $V_{m}\left(x ; b_{1}, b_{2}\right)=$ $E\left[D^{m}\right]$; using the representation

$$
M\left(x, y ; b_{1}, b_{2}\right)=1+\sum_{m=1}^{\infty} \frac{y^{m}}{m !} V_{m}\left(x ; b_{1}, b_{2}\right)
$$

and equating the coefficients of $y^{m}$ in (53) yield the ordinary differential equations (66) and (67).

Remark 8. When $m=1$, we get $V_{1}\left(x ; b_{1}, b_{2}\right)=V\left(x ; b_{1}, b_{2}\right)$.

\section{Conflict of Interests}

The authors declare that there is no conflict of interests regarding the publication of this paper.

\section{Acknowledgments}

The authors would like to thank the editor and the anonymous referees for their helpful comments, which have led to this improved version of the paper. The research was supported by the National Natural Science Foundation of China (no. 11171179), the Research Fund for the Doctoral Program of Higher Education of China (no. 20133705110002), and the Program for Scientific Research Innovation Team in Colleges and Universities of Shandong Province.

\section{References}

[1] B. de Finetti, "Su un'impostazione alternativa della teoria collettiva del rischio," in Proceedings of the transactions of the 15th International Congress of Actuaries, vol. 2, pp. 433-443, 1957.

[2] S. Asmussen and M. Taksar, "Controlled diffusion models for optimal dividend pay-out," Insurance, vol. 20, no. 1, pp. 1-15, 1997.

[3] H. Albrecher, R. Kainhofer, and R. F. Tichy, "Simulation methods in ruin models with non-linear dividend barriers," Mathematics and Computers in Simulation, vol. 62, pp. 277-287, 2003.

[4] H. Gao and C. Yin, "A perturbed risk process compounded by a geometric Brownian motion with a dividend barrier strategy," Applied Mathematics and Computation, vol. 205, no. 1, pp. 454464, 2008.
[5] H. U. Gerber and E. S. W. Shiu, "On the time value of ruin," North American Actuarial Journal, vol. 2, no. 1, pp. 48-78, 1998.

[6] H. U. Gerber and E. S. W. Shiu, "Optimal dividends: analysis with Brownian motion," North American Actuarial Journal, vol. 8, no. 1, pp. 1-20, 2004.

[7] N. Wan, "Dividend payments with a threshold strategy in the compound Poisson risk model perturbed by diffusion," Insurance, vol. 40, no. 3, pp. 509-523, 2007.

[8] H. U. Gerber and E. S. W. Shiu, "On optimal dividend strategies in the compound Poisson model," North American Actuarial Journal, vol. 10, no. 2, pp. 76-93, 2006.

[9] J. Cai, H. U. Gerber, and H. Yang, "Optimal dividends in an Ornstein-Uhlenbeck type model with credit and debit interest," North American Actuarial Journal, vol. 10, no. 2, pp. 94-119, 2006.

[10] H. Gao and C. Yin, "The perturbed Sparre Andersen model with a threshold dividend strategy," Journal of Computational and Applied Mathematics, vol. 220, no. 1-2, pp. 394-408, 2008.

[11] H. Yang and Z. Zhang, "On a perturbed Sparre Andersen risk model with multi-layer dividend strategy," Journal of Computational and Applied Mathematics, vol. 232, no. 2, pp. 612-624, 2009.

[12] H. Yang and Z. Zhang, "The perturbed compound Poisson risk model with multi-layer dividend strategy," Statistics \& Probability Letters, vol. 79, no. 1, pp. 70-78, 2009.

[13] Y. Fang and R. Wu, "Optimal dividends in the Brownian motion risk model with interest," Journal of Computational and Applied Mathematics, vol. 229, no. 1, pp. 145-151, 2009.

[14] H. Y. Wong and J. Zhao, "Optimal dividends and bankruptcy procedures: analysis of the Ornstein-Uhlenbeck process," Journal of Computational and Applied Mathematics, vol. 236, no. 2, pp. 150-166, 2011.

[15] C. Yin and Y. Wen, "An extension of Paulsen-Gjessing's risk model with stochastic return on investments," Insurance, vol. 52, no. 3, pp. 469-476, 2013.

[16] L. Breiman, Probability, Addison-Wesley, Reading, Mass, USA, 1968.

[17] P. Li, C. C. Yin, and M. Zhou, "The exit time and the dividend value function for one-dimensional diffusionn processes," Abstract and Applied Analysis, vol. 2013, Article ID 675202, 9 pages, 2013. 


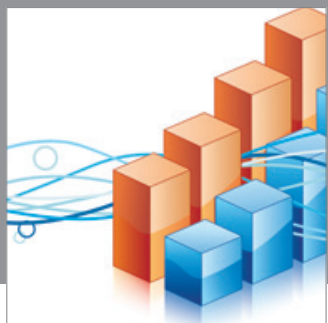

Advances in

Operations Research

mansans

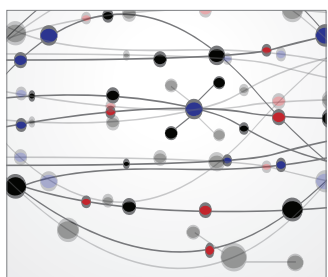

The Scientific World Journal
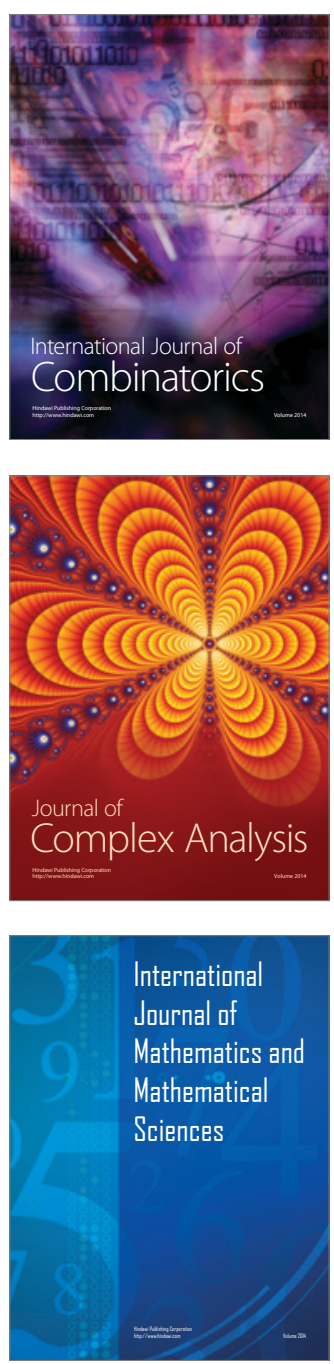
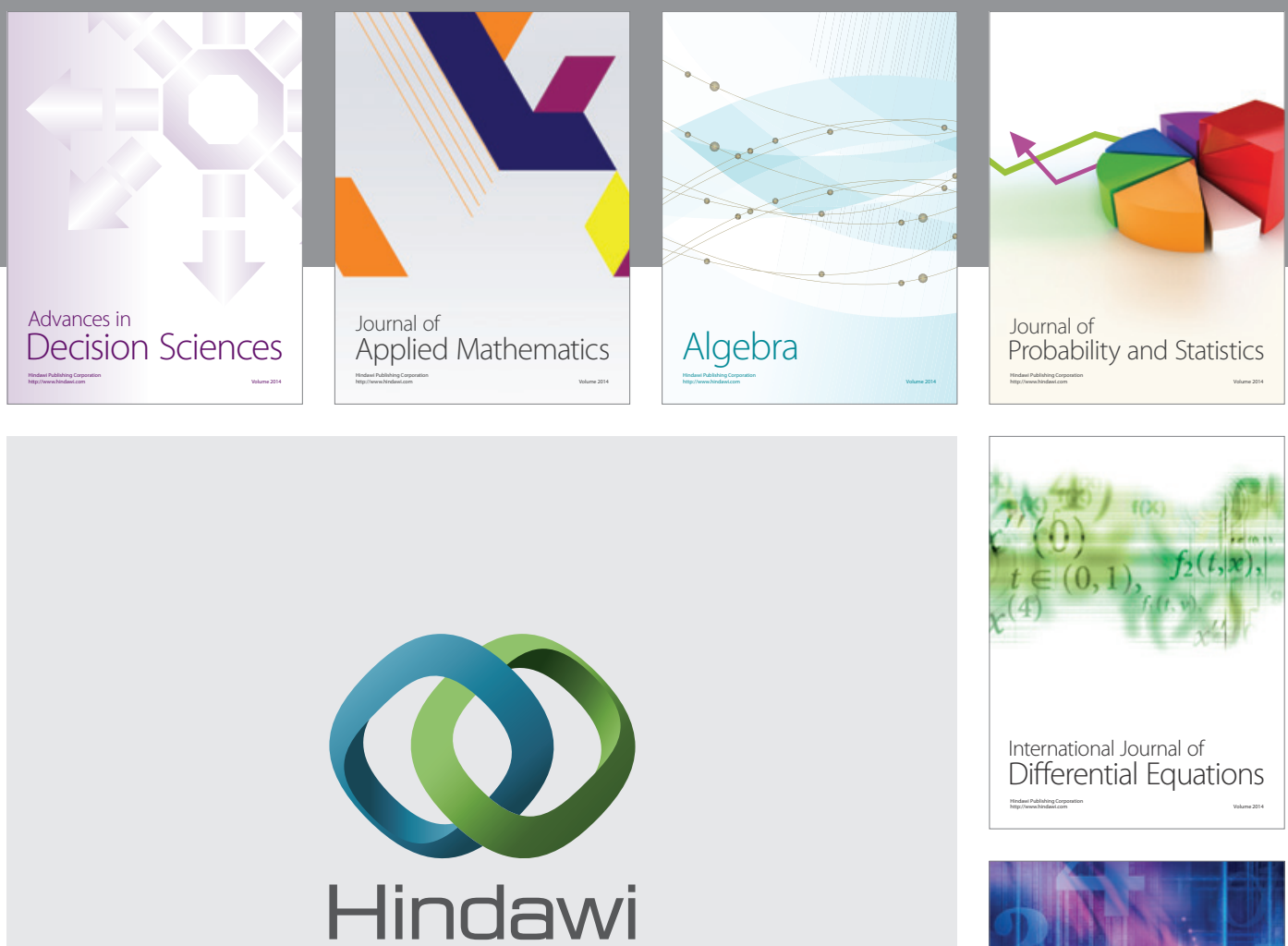

Submit your manuscripts at http://www.hindawi.com
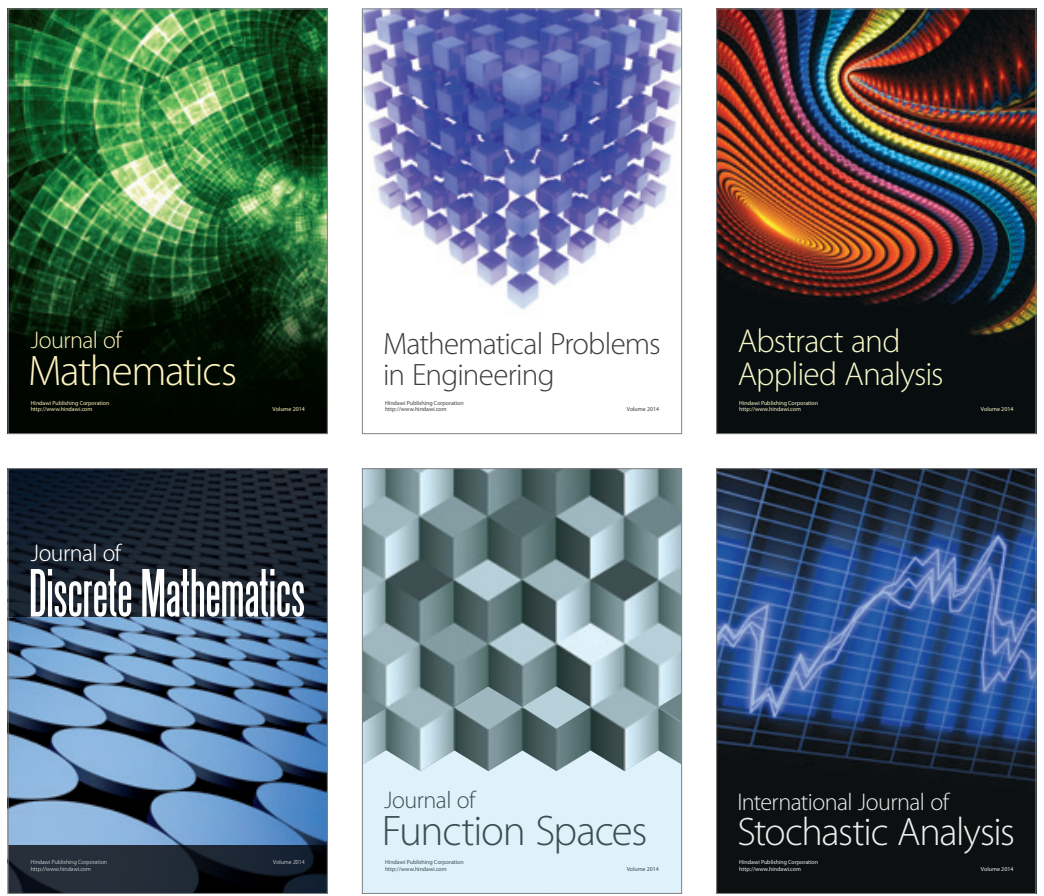

Journal of

Function Spaces

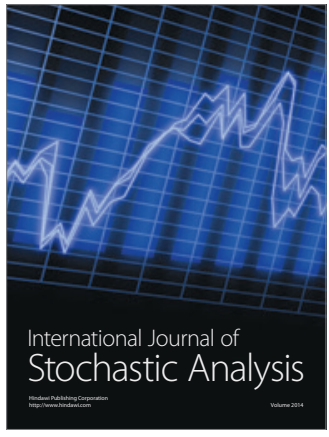

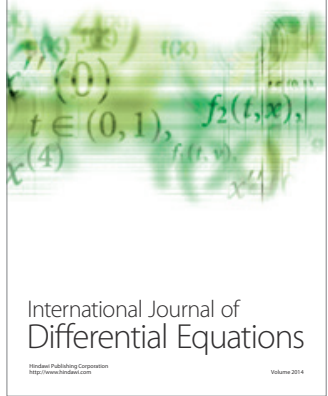
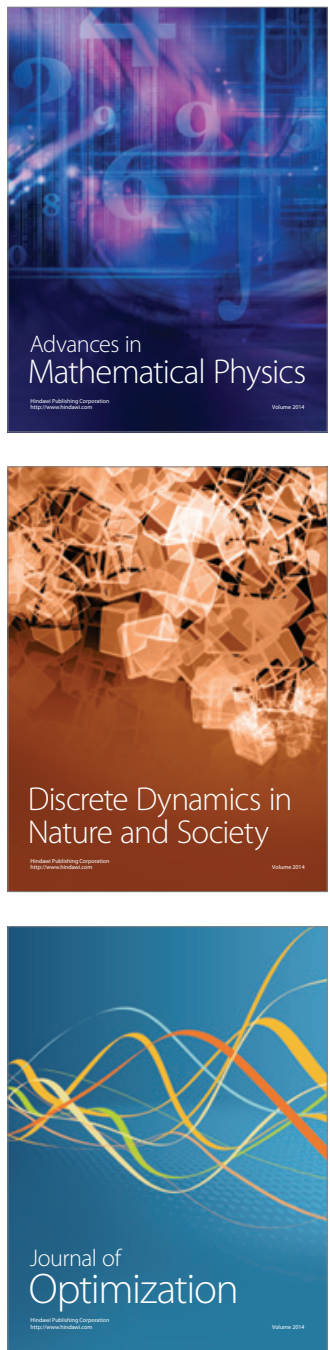